Virtual Mentor. January 2005, Volume 7, Number 1.

doi: 10.1001/virtualmentor.2005.7.1.ccas5-0501

Module 2

\title{
Case 2.1: Respect for Patient Decision Making-Mr. Douglas's Choice of Treatment
}

\section{Case Presentation}

Ian Douglas, a 53-year-old businessman, worked long hours and enjoyed a successful and lucrative career. He had smoked on and off for 25 years. Recently, Mr. Douglas had been feeling tired and having trouble breathing when he climbed stairs. Every so often he had chest pains. When Mr. Douglas and his wife, Lynn, sat down to talk to their teenage son about failing 2 of his classes. Mr. Douglas lost his temper and the chest pains started. This time they crept up the side of his neck, so he stopped and went to lie down. That's when he decided he was going to see a doctor.

In Dr. Kim's office, Mr. Douglas explained that he was having chest pains that lasted no longer than 5 minutes and were always relieved by rest. He said he'd been having the symptoms for a while, but they didn't seem to be getting any better or worse. Dr. Kim got an EKG in the office, which was normal, and had him undergo a stress test. Mr. Douglas had to stop the test because he started feeling fatigued and having mild chest pains. Dr. Kim explained that the tests showed some ischemia and marked ST changes on his EKG, indicating that Mr. Douglas needed an angiogram in the next day or 2 to better evaluate his coronary arteries.

When Mr. Douglas returned to the doctor for the results of the angiogram, Dr. Kim told him that his three main coronary arteries were all severely blocked. "The good news," Dr. Kim said, " is that we caught this before you had a major heart attack. The bad news is that you should have triple bypass surgery as soon as possible."

Mr. Douglas gasped. "Don't you think that's jumping the gun, I just have a little heart problem, right? I know I've got to do something about this. I'll quit smoking, and I'll go to the gym, but I'm not having open heart surgery for these little chest pains."

Dr. Kim shook his head, "Mr. Douglas, I understand that you believe your symptoms don't warrant major surgery, but your heart is working overtime right now. It is just a matter of time before it gives out. You should start eating better and you must stop smoking, but there is no question that the surgery is necessary, and I would like it to happen as soon as possible. You'll have to take a few weeks off work to recover from the procedure, but you should be able to return to work in a month or two."

"Listen, doc, I have a major deal that has to go through within the next month," Mr. Douglas insisted. "How about I start taking care of myself, and you let me get this deal signed, and then we'll talk about the surgery, okay?"

\section{What should Dr. Kim do? (select an option)}
A. Ask Mr. Douglas to restate his understanding of his medical condition and explain that lifestyle changes will not reverse the current condition.
B. Tell Mr. Douglas that he will die without surgery.
C. Ask Mr. Douglas to inform his wife of his condition so they can discuss his alternatives.
D. Recommend that Mr. Douglas get a second opinion. 
Virtual Mentor. January 2005, Volume 7, Number 1.

doi: 10.1001/virtualmentor.2005.7.1.ccas5a-0501

Module 2

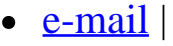

\section{Case 2.1: Respect for Patient Decision Making-Mr. Douglas's Choice of Treatment}

\section{Option Assessment}

A. Asking Mr. Douglas to restate the nature of his medical condition and explaining the role of lifestyle changes is preferable; it is supported by the first sentence of Opinion 8.08, "Informed Consent": "The patient's right of selfdecision can be effectively exercised only if the patient possesses enough information to enable an intelligent choice." By asking Mr. Douglas to restate the nature of his medical condition, the physician can discover whether he has the appropriate information. Moreover, explaining in detail the effectiveness of lifestyle choices (eg, quitting smoking and regular exercise) for prevention of his condition versus treatment of his condition is supported by the same sentence of Opinion 8.08, "Informed Consent": "The patient's right of self-decision can be effectively exercised only if the patient possesses enough information to enable an intelligent choice." By explaining (or re-explaining) these differences in effectiveness, the physician ensures that Mr. Douglas has enough information to make an informed decision.

B. Telling Mr. Douglas that he will die without surgery should be avoided because it is not supported by the Code and may violate Opinion 8.08, "Informed Consent": "The physician's obligation is to present the medical facts accurately to the patient....and to make recommendations for management in accordance with good medical practice." While it is likely that Mr. Douglas will have a heart attack if he doesn't have surgery, it is not certain that he will die. Telling the patient he will die is an attempt to coerce him into accepting the physician's recommended treatment; this constitutes undesirable paternalistic behavior on the physician's part.

C. Suggesting that Mr. Douglas inform his wife of his condition so they can discuss his alternatives is an acceptable option not clearly addressed by the Code. This course of action may fall under Opinion 8.08, "Informed Consent", which states that the "physician has an ethical obligation to help the patient make choices from among the therapeutic alternatives consistent with good medical practice." By encouraging Mr. Douglas to involve his spouse in the decision-making process, the physician acknowledges the value of (usually helpful) input from family and other social relationships in important decisions.

D. Suggesting that Mr. Douglas obtain a second opinion is an acceptable alternative not clearly addressed by the Code. This course of action may fall under Opinion 8.08, "Informed Consent," which states that the "physician has an ethical obligation to help the patient make choices from among the therapeutic alternatives consistent with good medical practice." A second opinion will likely emphasize to Mr. Douglas the seriousness of his condition and may help him make a reasoned decision. 
Virtual Mentor. January 2005, Volume 7, Number 1.

doi: 10.1001/virtualmentor.2005.7.1.ccas5b-0501

Module 2

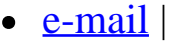

\section{Case 2.1: Respect for Patient Decision Making-Mr. Douglas's Choice of Treatment}

\section{Option Comparison}

To assure that Mr. Douglas is aware of the likely outcomes of his choice, option A is preferable and is expressly supported by the Code because it addresses the potential shortcoming of Mr. Douglas's decision-making capacity, specifically, his understanding of the likely consequence of no treatment. In requiring Mr. Douglas to restate his medical condition, option A gives Dr. Kim the chance to explain the limits of lifestyle options on treating existing conditions and to correct any of Mr. Douglas's misconceptions.

Option C, asking Mr. Douglas to bring his wife into the conversation, gives him an opportunity to carefully consider how his decision will affect the people and life he values. Option D (getting a second opinion) is also an acceptable alternative that gives Mr. Douglas another opportunity to have the severity of his condition explained. Although this may not help him clarify his values, it may emphasize to him the severity of his condition.

Options B, telling Mr. Douglas he will die, should be avoided. This options ignores the uncertainty inherent in any medical prognosis. No physician can know that a patient in Mr. Douglas's situation will die without treatment in the next month, and stating so attempts to coerce Mr. Douglas.

Preferable: Option A

Acceptable: Option C and D

Avoid: Option B

\section{Additional discussion and information}

The people and events in this case are fictional. Resemblance to real events or to names of people, living or dead, is entirely coincidental. The viewpoints expressed on this site are those of the authors and do not necessarily reflect the views and policies of the AMA.

(C) 2005 American Medical Association. All Rights Reserved. 
Virtual Mentor. January 2005, Volume 7, Number 1.

doi: 10.1001/virtualmentor.2005.7.1.ccas5c-0501

Module 2

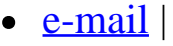

\section{Case 2.1: Respect for Patient Decision Making-Mr. Douglas's Choice of Treatment}

\section{Additional Information}

The right of patients to make informed decisions about their medical treatment is a cornerstone of contemporary ethical medical practice. The current Code reflects this right in a number of Opinions including Opinion 10.01, "Fundamental Elements of the Patient-Physician Relationship": "The patient has the right to receive information from physicians and to discuss the benefits, risks, and costs of appropriate treatment alternatives...[and] patients may accept or refuse any recommended medical treatment"; and Opinion 8.12, "Patient Information": "Patients have a right to know their past and present medical status and to be free of any mistaken beliefs concerning their conditions."

Generally, patients are not privy to the specialized knowledge of medicine, and, without help, they are likely to misunderstand their diagnoses and treatment options. Accordingly, physicians and other medical professionals are obliged to use their expertise to help patients make informed decisions about their medical care. As the Code emphasizes again and again, autonomous decision making is a fundamental tenet of good medical care. This point is expressed most directly in Opinion 8.08, "Informed Consent":

\section{Opinion 8.08, "Informed Consent"}

The patient's right of self-decision can be effectively exercised only if the patient possesses enough information to enable an intelligent choice...The physician's obligation is to present the medical facts accurately...[and] to help the patient make choices from among the therapeutic alternatives consistent with good medical practice...Social policy does not accept the paternalistic view that the physician may remain silent because divulgence might prompt the patient to forego needed therapy. Rational, informed patients should not be expected to act uniformly, even under similar circumstances, in agreeing to or refusing treatment.

For patients to exercise informed consent (to be autonomous) they must be competent and have decision-making capacity. Determination of a patient's decision-making capacity is an informal judgment by physicians and other health care practitioners about the patient's ability to give informed consent for this decision.

The following generally accepted elements of informed consent can help determine whether Mr. Douglas can give informed consent in this case: (1) the patient must be able to understand the information that he or she receives about his or her medical condition and the likely outcomes associated with all treatment options, including no treatment. (2) The patient must be informed of diagnosis and treatment alternatives with associated risks and benefits (including the risks and benefits of no treatment). (3) The patient must be able to make an uncoerced judgment about the information in light of his or her personal values and goals. Here it is recognized that an individual's "voluntariness," that is, his or her ability to make uncoerced decisions, can be compromised by physical, emotional, or psychological states and by the influence of drugs or alcohol. (4) The patient must be able to communicate his or her wishes with consistency over time. 
Mr. Douglas would undoubtedly be judged legally competent, but Dr. Kim has a legitimate concern about whether Mr. Douglas understands the likely consequences of his decision. He seems to know that the surgery will severely limit his activity for some time, but he may not be clear on the likely outcome of refusing surgery.

\section{$\underline{\text { Next Case }}$}

\section{Module 2 Table of Contents}

The people and events in this case are fictional. Resemblance to real events or to names of people, living or dead, is entirely coincidental. The viewpoints expressed on this site are those of the authors and do not necessarily reflect the views and policies of the AMA.

(C) 2005 American Medical Association. All Rights Reserved. 